\title{
The importance of IgG4 in the predictive model of thyroiditis
}

\author{
Milena S Pandrc ${ }^{1}$, Stanko Petrović ${ }^{2}$, Vanja Kostovski ${ }^{3}$, Marijana Petrović ${ }^{4}$ \\ and Miloš Zarićs
}

Departments of ${ }^{1}$ Internal Medicine, ${ }^{2}$ Gastroenterology, ${ }^{3}$ Clinic for Thoracic Surgery, ${ }^{4}$ Nephrology and ${ }^{5}$ Pathology, Militar Medical Academy, Crnotravska 17, 11000 Belgrade, Serbia
Correspondence

should be addressed

to M S Pandrc

Email

pandrcmilena@yahoo.com

\section{Summary}

Immunoglobulin (Ig)G4-related sclerosing disease (IgG4-RSD) is a new disease entity first proposed with regard to autoimmune pancreatitis. A 67-year-old male patient was examined because of weight loss and an abdominal pain. Based on the clinical characteristics, laboratory parameters and ultrasound features, we identified the diagnosis of the IgG4-related systemic disease (IgG4-RSD), that was confirmed by the histopathological analysis after the biopsy of the head of pancreas. After confirmation, we started with the corticosteroid therapy with a good clinical, biochemical and morphological response. During the previous therapy, the disturbance of glucoregulation appeared, so we had to change the modality of treatment. We decided to add Azathioprine to the therapy in a dose of $150 \mathrm{mg} /$ day. We achieved a stable phase of the disease with IgG $4.37 \mathrm{~g} / \mathrm{l}$ and IgG4 $0.179 \mathrm{~g} / \mathrm{l}$, and with no side effects from the therapy.

\section{Learning points:}

- There are potential clinical applications of identifying subsets of patients with IgG4 thyroiditis (FVHT and Riedel thyroiditis).

- A trial of immunosuppressive therapy should be included if a resection is deemed inadvisable.

- In particular, cases of FVHT that mimic malignancy, tissue and serum IgG4 may provide supportive diagnostic information.

\section{Background}

Recent studies present the unique subtype of Hashimoto's thyroiditis (HT), called immunoglobulin (Ig) G4 thyroiditis, characterized by lymphoplasmacytic inflammation, fibrosis (frequently in a storiform pattern), obliterative phlebitis, increased numbers of IgG4 positive plasma cells and elevated IgG4:IgG ratios. IgG4 thyroiditis cases are associated with male gender, rapid progression, subclinical hypothyroidism, more diffuse low echogenicity and higher levels of circulating antithyroid antibodies (1).

In the past few years, there have been numerous reports about what is currently termed immunoglobulin (Ig)G4-related sclerosing disease (IgG4-RSD), which is a new disease entity first proposed with regard to autoimmune pancreatitis. This syndrome is characterized by mass-forming lesions in various organs due to severe lymphoplasmacytic infiltrates and stromal fibrosis, hypergammaglobulinemia with a predominant increase in the IgG4 levels and alleviation of symptoms after steroid therapy (2).

\section{Case presentation}

A 67-year-old male patient was examined by a gastroenterologist due to weight loss and an abdominal pain. 


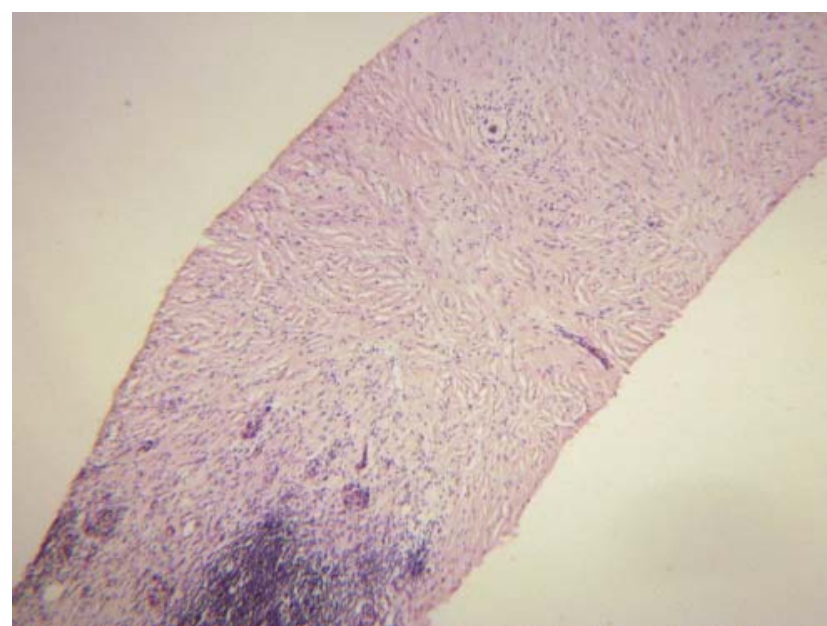

Figure 1

Dense lymphoplasmacytic infiltration and storiform fibrosis (HE staining, $4 \times$ ).

\section{Investigation}

Blood analysis was done and we registered a discreet elevation of biochemical markers of inflammation (SE: $57 \mathrm{~mm} / \mathrm{h}$, CRP: $5.0 \mathrm{mg} / \mathrm{l}$, fibrinogen: $4.8 \mathrm{~g} / \mathrm{l}$ ), anemia syndrome $\left(\mathrm{Er}=4.06 \times 10^{12} / \mathrm{l}, \mathrm{Hgb}=116 \mathrm{~g} / \mathrm{l}, \mathrm{Hct}=0.34 \mathrm{l} / \mathrm{l}\right)$, an elevation of the lactate dehydrogenase $(\mathrm{LDH}=408 \mathrm{U} / \mathrm{l})$, with hyperamylasemia - $749 \mathrm{U} / 1$. Hormonal examination revealed normal levels of parathyroid hormone $(\mathrm{PTH}=32.7 \mathrm{ng} / \mathrm{l}$ ), with subclinical hypothyroidism (freethyroxine $=1.42 \mathrm{ng} / \mathrm{dl}$, tri-iodothyronine $=1.66 \mathrm{ng} / \mathrm{dl}$, thyrotropin $=6.9 \mathrm{mU} / \mathrm{l}$ with an elevation of the anti TPO $\mathrm{ab}$ and anti $\mathrm{Tg} \mathrm{ab}$ ). The electrophoresis serum proteins pointed out the pick of IgG with extremely elevated IgG4 (IgG4 9.8 g/l, IgG4/IgG > 30\%). The thyroid's ultrasound revealed a polynodal gland, discreetly hypoechogenic, but of normal size. The abdominal ultrasound showed oversized hypoechogenic pancreas - so-called 'sausage-like pancreas.' Based on the clinical characteristics, the laboratory parameters and the ultrasound features, a differential diagnosis of IgG4-related systemic disease (IgG4-RSD) was made, confirmed by histopathological analysis after the biopsy of the head of pancreas, which showed lymphoplasmacytic infiltration with the immunohistochemistry's evaluation of more than $2 / 3$ lymphocytes IgG $4+/ \mathrm{HPF}$, and storiform fibrosis (Figs 1 and 2).

\section{Treatment}

After the confirmation of the diagnosis, we proceeded with the corticosteroid therapy, with a good clinical, biochemical and morphological response. During the previous therapy, the disturbance of glucoregulation appeared, so we had to change the modality of treatment. We decided to include Azathioprine in the therapy, at a dose of $150 \mathrm{mg} /$ day.

\section{Outcome and follow-up}

We achieved remission of the disease, with IgG $4.37 \mathrm{~g} / \mathrm{l}$, and IgG4 $0.179 \mathrm{~g} / \mathrm{l}$, and with no side effects from the therapy.

\section{Discussion}

Autoimmune pancreatitis (AIP) is now considered to encompass two separate disorders: type 1 AIP, which is associated with IgG4-related disease, and type 2 AIP, which has substantial clinical overlap with type 1 but distinctive pathological features. Type 1 AIP is currently diagnosed on the basis of a combination of clinical, laboratory and imaging studies (3).

The Japanese revised 'Clinical Diagnostic Criteria of Autoimmune Pancreatitis' (2006) contained three items: radiological imaging showing diffuse or segmental narrowing of the main pancreatic duct, and diffuse or segmental enlargement of the pancreas; laboratory data demonstrating abnormally elevated levels of serum gamma globulin IgG or IgG4, or presence of autoantibodies; and histological examination of the pancreas revealing lymphoplasmacytic infiltration and fibrosis. The presence of the imaging criterion is essential for the

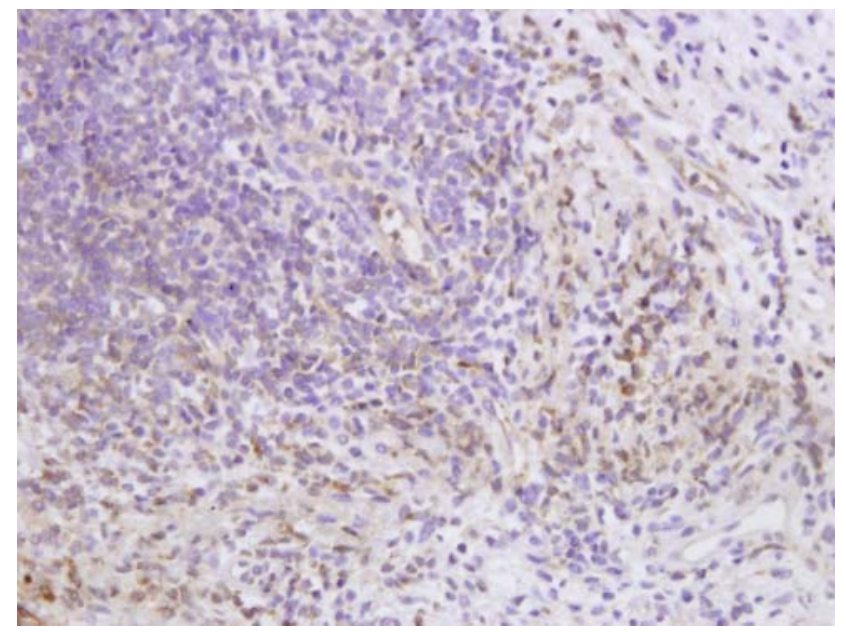

Figure 2

Infiltration of IgG4-positive plasma cells (IgG4 immunostaining, $20 \times$ ). 
diagnosis of AIP, together with either criterion 2 or criterion 3. These criteria are based on the minimum consensus of AIP to avoid misdiagnosing pancreatic cancer as far as possible (4).

AIP patients typically show diffuse enlargement of the pancreas, the so-called 'sausage-like' appearance, but segmental-type AIP is difficult to differentiate from pancreatic cancer (3).

The characteristic histological findings for the pancreas in AIP patients are known as lymphoplasmacytic sclerosing pancreatitis (LPSP), comprising dense infiltration of T lymphocytes and IgG4-positive plasma cells and storiform fibrosis. Obliterative phlebitis, representing obliteration of veins by the inflammatory process, is frequently detected and is a diagnostic feature of LPSP. Although these characteristic histological findings of AIP form a gold standard for diagnosing AIP, diagnosing AIP on biopsy or endoscopic ultrasound-guided fine needle aspiration (EUS-FNA) biopsy is sometimes difficult due to the small size of the sample obtained (5).

Most cases of AIP in Asia are type 1. Clinically, type 2 patients are around a decade younger than type 1 patients and are less likely to show elevated serum IgG4 levels. Type 2 AIP rarely has sclerosing extrapancreatic lesions, but is sometimes associated with acute pancreatitis and ulcerative colitis, which are rarely associated in type 1 AIP patients. Type 2 is also responsive to steroid therapy. Types 1 and 2 AIP are clinically, regionally and ethnically different entities, sharing the need for accurate differentiation from pancreatic cancer (3).

Other clinical characteristics of type 1 include preponderance toward elderly men, common initial symptoms of obstructive jaundice, and favorable response to steroid therapy (6).

These histologic features have been utilized as morphological hallmarks of IgG4-RSD.

Apart from histology, the elevated serum IgG4 level is used as a clinical clue for suspected AIP and other IgG4related lesions. Currently, the upper limit of normal for serum IgG4 is taken to be $140 \mathrm{mg} / \mathrm{dl}$ according to the Mayo Clinic criteria, $135 \mathrm{mg} / \mathrm{dl}$ according to the Asian Criteria, whereas some use a cut-off of $130 \mathrm{mg} / \mathrm{dl}$ (7).

However, $\sim 7-10 \%$ of patients with pancreatic cancer and $10 \%$ of patients with cholangiocarcinoma have elevated serum IgG4 levels. Therefore, serum IgG4 elevation alone should not be used to make the diagnosis of AIP or IgG4-RSD (1).

Now that the concept of IgG4-RD has been established, a precise definition of elevated serum IgG4 levels is required. The present findings indicated that high serum
IgG4 concentrations are not specific to IgG4-RD. The optimal cutoff value in some studies was $144 \mathrm{mg} / \mathrm{dl}$ when compared with rheumatic, allergic and common diseases. This value was determined at 90\% sensitivity and specificity, and together with the AUC it was useful for diagnosis. Therefore, in the daily clinic, the usual cutoff value of $135 \mathrm{mg} / \mathrm{dl}$ in AIP does not cause any problems, and it is also useful for diagnosing any IgG4-RD (7).

Typical AIP shows diffuse changes of the pancreas, but several cases have shown segmental changes. The current concept of AIP, including associated extrapancreatic lesions, suggests that AIP may represent a systemic disease (4) (8).

Recognition of the IgG4-RSD entities does not just contribute to the pathological re-classification of preexisting diseases, but also helps clinicians guide the identification and treatment of the disease. Because many of these lesions are present as a mass that is clinically suspicious for malignant disease causing unnecessary operation, they are nevertheless effectively responsive to steroid therapy (9).

It is important to be able to differentiate IgG4-RD from cancer, because IgG4-RD presents with mass-forming lesions (10).

About 25\% of patients with AIP show biochemical evidence of hypothyroidism, often accompanied by TgAb. On the basis of immunohistochemistry of IgG4 and IgG, we originally described two subtypes of HT: i) IgG4 thyroiditis (IgG4 related, IgG4-positive plasma cell-rich group) and ii) non-IgG4 thyroiditis (non-IgG4 related, IgG4-positive plasma cell-poor group).

Although the cut-off value of IgG4-positive plasma cell has not been standardized, we adopted $20 \%$ IgG4-positive plasma cells and 30\% IgG4/IgG ratio as the diagnostic threshold, because it has high specificity and sensitivity for defining a significant increase of IgG4-positive plasma cells in thyroid tissue (11).

IgG4 thyroiditis reveals significantly more association with subclinical hypothyroidism. A laboratory examination further reveals that preoperative serum titers of thyroid autoantibodies, both TgAb and TPOAb, are significantly higher in the IgG4 thyroiditis group than the non-IgG4 thyroiditis group, which also supports their different degrees of parenchyma destruction on histologic specimens. Finally, a sonographic examination shows that IgG4 thyroiditis is significantly correlated with diffuse low echogenicity, while in non-IgG4 thyroiditis diffuse coarse echogenicity is more often noted. Serum IgG4 levels could represent the status of IgG4-positive plasma cell infiltration in thyroid tissue (1).

Our patient had the characteristic findings that can be summarized clinically, radiologically, serologically and 
histopathologically as follows: preponderance toward elderly men; weight loss and abdominal pain as frequent initial symptoms; the association with impaired pancreatic endocrine function and an extrapancreatic lesion; favorable response to steroid therapy; radiological finding of enlargement of the pancreas; serological findings of elevated serum IgG4 levels, along with the presence of some autoantibodies; and characteristic histopathological findings of dense lymphoplasmacytic infiltration with fibrosis, without obliterative phlebitis. The present findings indicated that high serum IgG4 concentrations are not specific to IgG4-RD. However, 7-10\% of patients with pancreatic cancer and $10 \%$ of patients with cholangiocarcinoma have elevated serum IgG4 levels. Therefore, serum IgG4 elevation alone should not be used to make the diagnosis of AIP or IgG4-RSD (1).

In practice, neither ducts nor veins are seen commonly in needle biopsies. In that case, the cell-rich storiform fibrosis becomes a critical finding in the immunohistochemistry of IgG4. Increased numbers of IgG4positive plasma cells (more than 10/hpf), particularly if they are diffuse, strongly support the diagnosis of AIP. Some studies have shown that fine needle aspiration of the pancreas can provide sufficient diagnostic material in this clinical setting. This diagnosis depends on recognizing stromal fragments in cytology preparations (5).

It is also important to distinguish IgG4-RD from rheumatic diseases because IgG4-RD can present with hypergammaglobulinemia, hypocomplementemia, elevated circulating immune complexes and positive rheumatoid factor. Our patient had no ocular symptoms or signs (negative Shirmer's test), oral symptoms or normal values of antibodies:anti Ro/SS-A antibodies ( $2.7 \mathrm{U} / \mathrm{ml})$, anti La/SS-B antibodies $(<0.1 \mathrm{U} / \mathrm{ml})$, so we excluded Sjögren's syndrome.

\section{Declaration of interest}

The authors declare that there is no conflict of interest that could be perceived as prejudicing the impartiality of the research reported.

\section{Funding}

This research did not receive any specific grant from any funding agency in the public, commercial or not-for-profit sector.

\section{Patient consent}

The written informed consent has been obtained from the patient for publication of the submitted article and accompanying images by their signature of our consent form that is scanned and sent together with other files.

\section{Author contribution statement}

Each author contributed according to his field of interest. All authors checked the literature and together made the protocol for treatment.

\section{References}

1 Kakudo K, Li Y, Hirokawa M \& Ozaki T 2011 Diagnosis of Hashimoto's thyroiditis and IgG4-related sclerosing disease. Pathology International 61 175-183. (doi:10.1111/j.1440-1827.2011.02661.x)

2 Sato Y, Notohara K, Kojima M, Takata K, Masaki Y \& Yoshino T 2010 IgG4-related disease: historical overview and pathology of hematological disorders. Pathology International 60 247-258. (doi:10.1111/ j.1440-1827.2010.02524.x)

3 Kensuke T, Terumi K \& Yoshinori I 2011 Autoimmune pancreatitis and IgG4-related sclerosing cholangitis. Current Opinion in Rheumatology 23 80-87. (doi:10.1097/BOR.0b013e3283412f60)

4 Okazaki K, Kawa S, Kamisawa T, Naruse S, Tanaka S, Nishimori I, Ohara H, Ito T, Kiriyama S, Inui K et al. 2006 Clinical diagnostic criteria of autoimmune pancreatitis: revised proposal. Journal of Gastroenterology 41 626-631. (doi:10.1007/s00535-006-1868-0)

5 Detlefsen S, Drewes AM, Vyberg M \& Klöppel G 2009 Diagnosis of autoimmune pancreatitis by core needle biopsy: application of six microscopic criteria. Virchows Archiv 454 531-539. (doi:10.1007/ s00428-009-0747-5)

6 Khosroshahi A \& Stone JH 2011 A clinical overview of IgG4-related systemic disease. Current Opinion in Rheumatology 23 57-66. (doi:10.1097/BOR.0b013e3283418057)

7 Sah RP \& Chari ST 2011 Serologic issues in IgG4-related systemic disease and autoimmune pancreatitis. Current Opinion in Rheumatology 23 108-113. (doi:10.1097/BOR.0b013e3283413469)

8 Okazaki K, Kawa S, Kamisawa T, Ito T, Inui K, Irie H, Irisawa A, Kubo K, Notohara K, Hasebe O et al. 2009 Japanese clinical guidelines for autoimmune pancreatitis. Pancreas 38 849-866. (doi:10.1097/ MPA.0b013e3181b9ee1c)

9 Kalaitzakis E \& Webster GJ 2011 Review article: autoimmune pancreatitis - management of an emerging disease. Alimentary Pharmacology \& Therapeutics 33 291-303. (doi:10.1111/j.1365-2036. 2010.04526.x)

10 Yamamoto M, Tabeya T, Naishiro Y, Yajima H, Ishigami K, Shimizu Y, Obara M, Suzuki C, Yamashita K, Yamamoto H et al. 2012 Value of serum IgG4 in the diagnosis of IgG4-related disease and in differentiation from rheumatic diseases and other diseases. Modern Rheumatology 22 419-425. (doi:10.3109/s10165-011-0532-6)

11 Li Y, Bai Y, Liu Z, Ozaki T, Taniguchi E, Mori I, Nagayama K, Nakamura H \& Kakudo K 2009 Immunohistochemistry of IgG4 can help subclassify Hashimoto's autoimmune thyroiditis. Pathology International 59 636-641. (doi:10.1111/j.1440-1827.2009.02419.x)

Received in final form 9 June 2015

Accepted 17 June 2015 